\title{
SIRT1 Expression and BRAF V600E Mutation in Papillary Thyroid Cancer: Implications for Diagnosis and Prognosis
}

\section{Tong Li}

Peking Union Medical College Hospital

\section{Guangzhi Wang}

Second Hospital of Dalian Medical University

\section{Zhenlu Li}

Second Hospital of Dalian Medical University

\section{Ning Zhang}

Second Hospital of Dalian Medical University

\section{Yuguo Li}

Second Hospital of Dalian Medical University

\section{Yongfu Zhao}

Second Hospital of Dalian Medical university

Xiaofeng Tian ( $\nabla$ txfdl@dmu.edu.cn )

Second Hospital of Dalian Medical University https://orcid.org/0000-0002-3034-0634

\section{Research}

Keywords: papillary thyroid cancer; BRAF V600E mutation; SIRT1; metastasis; MAPK

Posted Date: May 13th, 2020

DOI: https://doi.org/10.21203/rs.3.rs-27747/v1

License: (c) (i) This work is licensed under a Creative Commons Attribution 4.0 International License. Read Full License 


\section{Abstract \\ Background}

The BRAF V600E mutation is closely associated with aggressive clinicopathologic features and increased recurrence and disease-specific mortality of papillary thyroid cancer (PTC). Silent mating type information regulation 2 homolog-1 (SIRT1) has long been suggested to have tumor promotor activity and is strongly associated with the presence of BRAF mutations and higher grades of malignancy in many cancer types. However, no evidence or mechanism have been identified in PTC.

\section{Methods}

PTC tissue samples and their adjacent normal tissues from 42 PTC patients were collected; qRT-PCR, western blotting, T1799A BRAF mutation testing were utilized and the association with clinicopathologic features were assessed. Three different thyroid cell lines, Nthy-ori $3-1$, TPC-1 and BCPAP, were used in in vitro studies. Cell counting kits and cell cycle analyses were utilized to investigate the level of cell proliferation and cell cycle progression. Transwell and Matrigel invasion assays were used to investigate the level of cell migration and invasion.

\section{Results}

SIRT1 is significantly upregulated in PTC, especially in lymph node metastatic and BRAF V600E-mutated patients. Cell counting kits and cell cycle analyses demonstrated that SIRT1 significantly promoted cell proliferation and cell cycle progression in BRAF V600E-mutated cells. Transwell and Matrigel invasion assays confirmed that SIRT1 promoted the migration and invasion ability of BRAF V600E-mutated cells. Additionally, the results of western blotting showed that the aberrant activation of the mitogen-activated protein kinase (MAPK) pathway played a crucial role in the tumor-promoting role of SIRT1 in BRAF V600E-mutated PTC biology.

\section{Conclusions}

SIRT1 expression with a BRAF V600E mutation may serve as new candidate targets for the diagnosis and treatment of papillary thyroid carcinomas.

\section{Introduction}

Thyroid cancer is the most common endocrine malignancy, and its global incidence has rapidly increased in recent decades (1). There has been a marked increase in the global age-standardized incidence of thyroid cancer by $20 \%$ between 1990 and 2013, and this was the highest increase among all cancers (2). Thyroid cancers with a follicular cell origin include papillary thyroid cancer (PTC), follicular thyroid cancer 
(FTC), poorly differentiated thyroid cancer (PDTC), and anaplastic thyroid cancer (ATC). PTC accounts for more than $80 \%$ of all thyroid cancer types and is the major component of the marked rise in the incidence of thyroid cancer. When diagnosed at an early stage, indolent PTC has a relatively optimistic prognosis with traditional therapies, including surgery, thyroid-stimulating hormone (TSH) suppression therapy, and radioactive iodine (RAI) therapy (3). However, traditional therapies have limited effects in aggressive and dedifferentiated papillary thyroid cancers (DePTCs) $(4,5)$. Therefore, it is significant to distinguish patients who need aggressive treatments to offer them a better prognosis. To date, there have been several clinicopathological risk factors, such as older age at diagnosis, extrathyroidal invasion, and distant metastasis, that are known to be associated with a higher risk of PTC prognosis (6). However, the lack of accuracy limits their general use in clinical practice.

Recently, many studies have shown a significant association of the BRAF V600E mutation with aggressive clinicopathological features and increased cancer-related mortality of PTC patients (7). The BRAF V600E mutation, the T1799A transverse point mutation of BRAF that results in a valine-to-glutamic acid change in codon 600 , which is the most common genetic alteration in PTC, was initially found in melanoma (8) and subsequently in other human cancers, including colorectal (9) and thyroid cancers (10). Studies have confirmed that the expression of the BRAF V600E mutant protein could constitutively activate the mitogen-activated protein kinase (MAPK) signaling pathway, which increases the recurrence and disease-specific mortality of PTC $(11,12)$. This mutation constitutively activates the BRAF kinase and subsequently the MAPK signaling pathway $(13,14)$. It has been widely confirmed that the BRAF V600E mutation is associated with increased recurrence and disease-specific mortality of PTC, as exemplified by the fact that BRAF V600E was identified to have a higher risk for recurrence in solitary intrathyroidal PTC larger than $2.0 \mathrm{~cm}$ and less than $4.0 \mathrm{~cm}(15)$.

SIRT1, an NAD-dependent protein deacetylase, is involved in diverse cellular processes, including stress response, longevity, and cancer (16-18). It has been reported that the overexpression of SIRT1 deacetylases p53, leading to the suppression of p53 activity and therefore may elevate the risk of cancer $(19,20)$. The upregulated expression of SIRT1 indicates poor prognosis of patients in a large number of primary solid tumor types (21-24). The inhibition of SIRT1 has been shown to be associated with inhibited cell proliferation and arrested cell cycle of PTC cells while being regulated by hypermethylated in cancer-1 (HIC-1) (25). Interestingly, higher expression of SIRT1 in colorectal cancer has been shown to be strongly associated with the presence of a BRAF mutation and higher grades of malignancy. In addition, studies on BRAF V600E-mutated melanoma cells also found that SIRT1 plays a crucial role in regulating cell proliferation and the senescence-like phenotype $(26,27)$. However, the relationship between SIRT1 and the BRAF V600E mutation in PTC biology remains to be elucidated. In this study, we assessed the expression levels of SIRT1, evaluated the BRAF V600E mutation status in PTC and performed in vitro studies to further elucidate its mechanism in PTC.

\section{Material And Methods}




\section{Patients, sample collection and immunohistochemistry (IHC)}

A total of 42 PTC surgical specimens of cancerous tissues and their paired adjacent nonneoplastic tissues were obtained from patients who underwent surgery between 2017 and 2018 at Second Hospital of Dalian Medical University. None of the patients received neoadjuvant therapy. Tumor staging used the 8th edition of the TNM system designed by the American Joint Committee on Cancer (AJCC). Rabbit antihuman SIRT1 monoclonal antibody (1:1000, \#9475, CST, Boston, USA) was used for the standard immunohistochemical staining procedures. The brown granules in the cytoplasm were considered positive staining. For the assessment of cytoplasmic staining, the staining intensity was scored as follows: negative (score 0), bordering (score 1), weak (score 2), moderate (score 3), and strong (score 4). The staining extent was graded into five parts according to the percentage of elevated staining cells in the field as follows: negative (score 0), 0-25\% (score 1), 26-50\% (score 2), 51-75\% (score 3), and 76$100 \%$ (score 4). SIRT1 expression was evaluated by combining the staining intensity and extent. All patients provided written informed consent for the use of their tumor tissues for clinical research, and the project protocols were approved by the Medical Ethics Committee of Second Hospital of Dalian Medical University.

\section{Total RNA extraction and qRTPCR}

RNA extraction was performed using TRIzol Reagent (\#15596026; Invitrogen, Carlsbad, CA, USA), and cDNA synthesis was performed in a TransScript All-in-One First-Strand cDNA Synthesis SuperMix for qPCR Kit (\#AT341, TransGen Biotech, Beijing, China). SIRT1 cDNAs were amplified using the following primers synthesized by Invitrogen (Shanghai, China): SIRT1: 5'-TGACGTGGACATCCGCAAAG-3' (forward) and 5'-CTGGGCACCTAGGACATCGA-3' (reverse). mRNA expression was detected with a TransStart Top Green qPCR SuperMix Kit (\#AQ131, TransGen Biotech) using an ABI 7500 Real-Time PCR System (Applied Biosystems, USA). B-actin was used as an endogenous control. Each sample was tested at least three times.

\section{Immunoblotting}

Cells and tissues were lysed using RIPA Lysis Buffer System (\#sc-24948; Santa Cruz Biotechnology, Santa Cruz, CA) with Phosphatase Inhibitor Cocktail 3 (\#P0044; Sigma-Aldrich, St Louis, MO, USA). Equal protein loads were separated using sodium dodecyl sulfate-polyacrylamide gel electrophoresis (SDSPAGE), transferred to a polyvinylidene fluoride (PVDF) membrane (Millipore, Billerica, MA, USA), and blocked with $5 \%$ nonfat milk in TBST buffer at room temperature for $2 \mathrm{~h}$. The membranes were incubated with primary antibodies at $4{ }^{\circ} \mathrm{C}$ overnight and then washed in TBST buffer. Horseradish peroxidase (HRP)-conjugated secondary antibodies were used for the subsequent incubation. Signals were visualized using the ECL Reagent (\#WBKLS0500; Millipore, Billerica, MA) by a LAS-4000 image analyzer (Fuji Photo Film Co., Tokyo, Japan). The data were normalized to GAPDH. The primary antibodies used were the following: anti-SIRT1 (\#9475, CST), anti-p-ERK1/2 (\#4370), anti-p-JNK (\#4668), anti-p-P38 (\#4511), anti-GAPDH (\#ab8245, Abcam, UK), anti-ERK1/2 (\#16443-1-AP, Proteintech, Wuhan, China), anti- 
JNK (\#51151-1-AP), anti-BAX (\#50599-2-AP), anti-BCL-2 (\#12789-1-AP), and anti-Cyclin D1 (\#60186-1AP). HRP-linked secondary antibodies used were the following: anti-mouse lgG (\#7076, CST) and antirabbit lgG (\#7074).

\section{BRAF V600E mutational analysis in PTC}

Genomic DNA was isolated and purified from formalin-fixed paraffin-embedded (FFPE) primary PTC tissue blocks using a QIAamp DNA FFPE Tissue Kit (\#56404; QIAGEN, Germany) according to the manufacturer's instructions. The genomic DNA was quantified using a Merinton SMA4000 spectrophotometer (Merinton Inc., Beijing, China). Human BRAF exon 15 was amplified using the following primers: 5'-ACACATTTCAAGCCCCAAAAA-3' (forward) and 5'-CAGCATCTCAGGGCCAAAAA-3' (reverse). The purified PCR products were then sequenced on an ABIPRISM $3730 \mathrm{XL}$ automated genetic analyzer (Life Technologies, Carlsbad, CA).

\section{Cell line and culture conditions}

Two human PTC cell lines BCPAP (BRAF V600E-mutated cell line) and TPC-1 (with wild-type BRAF) and one normal thyroid follicular epithelial cell line Nthy-ori $3-1$ were provided by the Institute of Interdisciplinary Research (Berkeley, CA). The cells were authenticated using short tandem repeat analyses (ABI 3730XL Genetic Analyzer, Life Technologies, MA) and tested for mycoplasma. TPC-1 and BCPAP cells were cultured in Ham's F-12K (Kaighn's) Medium (\#21127-022; Gibco, Grand Island, NY, USA), and Nthy-ori $3-1$ cells were cultured in Dulbecco's modified Eagle medium (\#11995-065) supplemented with 10\% heat-inactivated fetal bovine serum (FBS, \#12483-020; Gibco) and 1\% penicillin-streptomycin (\#15140-122). The cells were cultured at $37^{\circ} \mathrm{C}$ in a humidified incubator with $5 \% \mathrm{CO}_{2}$.

\section{RNA interference using siRNA}

SIRT1-specific small interfering RNA (siRNA) and a scrambled negative control siRNA (si-NC) were provided by GenePharma (Shanghai, China). The sequences of the siRNA were as follows: SIRT1, forward 5'-TGGAACAGGTTGCGGGAAT-3' and reverse 5'-CTGGGCACCTAGGACATCGA-3'. The cells were transfected at 70\% confluence using Lipofectamine 3000 Reagent (\#L3000015; Invitrogen, Carlsbad). They were then harvested $48 \mathrm{~h}$ after transfection for further analysis. At least three replicates were analyzed for all knockdown experiments.

\section{Overexpression of SIRT1}

The human SIRT1 CRISPR Activation Plasmid (\#sc-400085-ACT) and Control CRISPR Activation Plasmid (\#sc-437275) were obtained from Santa Cruz Biotechnology, Inc. (Santa Cruz, CA). TPC-1 and BCPAP cells were transfected using UltraCruz ${ }^{\circledR}$ Transfection Reagent (\#sc-395739) according to the manufacturer's instructions. PTC cells were then incubated for $48 \mathrm{~h}$ posttransfection.

\section{Cell cycle analysis}

Cell Cycle and Apoptosis Analysis Kit (\#C1052; Beyotime, Shanghai) was used to analyze the cell cycle distribution according to the manufacturer's protocol. Briefly, transfected PTC cells were seeded and fixed 
with $70 \%$ ethanol in 6-well plates at $4{ }^{\circ} \mathrm{C}$ overnight. The cells were washed twice and resuspended in phosphate buffered saline (PBS). Propidium iodide and ribonuclease were added to the PBS, and the cells were incubated for $30 \mathrm{~min}$ at $37^{\circ} \mathrm{C}$. A FACSCalibur flow cytometer (BD, USA) was used to detect the cell cycle. The experiment was repeated in triplicate, and each experiment had two specimens.

\section{Cell proliferation assay (CCK-8 assay)}

The transfected cells were seeded at $5 \times 10^{3}$ cells per well in 96-well plates and then incubated at $37^{\circ} \mathrm{C}$ for four consecutive days. A $10 \mu$ l aliquot of the Cell Counting Kit-8 reagent (Dojindo, Japan) was added to each well. After incubating for $2.5 \mathrm{~h}$, the absorbance was measured using a spectrophotometer (BioRad, USA) at $450 \mathrm{~nm}$ (OD450). All assays were repeated in triplicate.

\section{Apoptotic cell death assay}

Terminal deoxynucleotidyl transferase UTP nick-end labeling (TUNEL) staining was performed using a TUNEL Label Mix Kit from Roche (\#11767291910; Mannheim, Germany) according to the manufacturer's protocol. Briefly, the PFA-fixed cells were incubated with $0.1 \%$ Triton X-100 for 10 min. After washing with PBS, $50 \mu$ of the TUNEL reaction mixture was added, and the cells were incubated for $1 \mathrm{~h}$ at $37^{\circ} \mathrm{C}$. The cells were then washed, and the nuclei were counterstained with 4',6-diamidino-2-phenylindole (DAPI, \#D9542; Sigma-Aldrich, St Louis, MO). The green (TUNEL) / blue (DAPI)-stained slides were visualized under a fluorescence microscope (Olympus BX 51, Tokyo, Japan).

\section{Wound-healing assay}

Confluent monolayers of transfected TPC-1 and BCPAP cells were seeded and incubated in 6-well plates until they reached $95 \%$ confluence. Then, scratches (wounds) were performed with Eppendorf pipette tips. Serum-free media were used to rinse out the detached cells. The remaining cells were cultured and observed for another $24 \mathrm{~h}$. The migrating cells between the scratches were photographed, and the results were quantified and analyzed using ImageJ software.

\section{Matrigel Invasion Assay}

We utilized Transwell cell culture chambers with 8 um Pore Polycarbonate Membrane Insert (\#3422; Corning Incorporated, NY) and Matrigel (\#SPC-356234; BD Biosciences, Oxford, UK) to perform invasion assays. The upper chamber was seeded with $1 \times 10^{4}$ transfected cells with serum-free medium, while the lower chamber was filled with culture medium containing 10\% FBS as the chemoattractant. After $24 \mathrm{~h}$ of incubation, cells that had not invaded through the pores were manually wiped off using cotton swabs. Then, the successfully invaded cells were fixed with 4\% paraformaldehyde (PFA, \#P6148; Sigma-Aldrich, St Louis, MO) for 30 min and stained with $0.1 \%$ Crystal Violet Staining Solution (\#G1063; Beijing Solarbio Science \& Technology Co., Ltd., Beijing, China) for another $30 \mathrm{~min}$. The number of invaded cells was counted under a microscope.

\section{Statistical analysis}


Statistical analyses were performed using GraphPad Prism 6.0 (GraphPad Prism Software, San Diego, CA, USA). Differences between primary PTC tissues and matching adjacent normal tissues were analyzed by Wilcoxon signed rank sum test. The aberrant status of SIRT1 and BRAF V600E and their associations with clinicopathologic characteristics were evaluated by chi-square tests or Fisher's exact tests. Twotailed Student's $t$-tests and one-way ANOVA were adopted to compare results among the groups. All data are presented as the mean \pm standard deviation. Differences with a $P$-value less than 0.05 were interpreted as statistically significant. All experiments were repeated at least three times.

\section{Results}

\section{SIRT1 expression and BRAF V600E mutation status in PTC tissues and their correlations with clinicopathological features}

We examined the mRNA and protein expression of SIRT1 in primary PTC tissues and matched adjacent normal tissues (ANT). Significantly higher expression of SIRT1 was found in 23 of 42 (54.76\%) cancerous tissues compared with adjacent normal tissues (Fig. 1A and 1B). In addition, the correlation between SIRT1 levels and patients' clinicopathological characteristics was assessed to determine its clinical significance (Table 1). We found that the expression of SIRT1 was significantly correlated with lymph node metastasis $(p<0.05)$ and a BRAF V600E mutation $(p<0.01)$. SIRT1 expression was higher in $65.63 \%(21 / 32)$ of the lymph node metastatic patients and in $67.74 \%(21 / 31)$ of the BRAF V600Emutated patients. Furthermore, we also confirmed that the BRAF V600E mutation was significantly correlated with lymph node metastasis in PTC patients (Table 2).

\section{BRAF V600E-mutated PTC cells possessed the highest level of SIRT1 expression}

SIRT1 expression was also detected in two human PTC cell lines, TPC-1 (with wild-type BRAF) and BCPAP (with the BRAF V600E mutation) and one normal thyroid follicular epithelial cell line (Nthy-ori 3 - 1), using western blot analysis (Fig. 2). We found that TPC-1 and BCPAP cells exhibited a notable upregulation of SIRT1 compared with Nthy-ori $3-1$ cells $(p<0.05)$. In addition, BCPAP cells had significantly higher SIRT1 levels compared to TPC-1 cells $(p<0.05)$. Therefore, to assess the relationship between SIRT1 and the BRAF V600E mutation in PTC, we used the TPC-1 and BCPAP cell lines for further examination.

\section{SIRT1 promoted cell proliferation of BRAF V600E-mutated cells}

The siRNAs were used to downregulate endogenous SIRT1 expression in TPC-1 and BCPAP cells. For SIRT1 overexpression, we synthetized and transfected CRISPR RNA-guided activation of SIRT1 (CRISPR SIRT1) and their negative controls into the two cell lines. The knockdown or activation of SIRT1 in PTC cells resulted in efficient changes in SIRT1 levels, as confirmed by western blot analyses (Fig. 3A and 3B).

To assess the role of SIRT1 on the proliferation of PTC cells, we performed a CCK-8 assay on TPC-1 and BCPAP cells. After incubating for four days, we found that knocking down SIRT1 significantly inhibited the cell viability $(p<0.01)$ and that the CRISPR SIRT1-transfected BCPAP cells had a higher cell 
proliferation than that in the control group $(p<0.05)$ at $48 \mathrm{~h}$ (Fig. 3C). However, we failed to observe any significant variations in TPC-1 cells (Fig. 3D).

\section{SIRT1 facilitated the cell cycle progression in BRAF V600E-mutated cells}

To determine whether the effect of SIRT1 on cell proliferation was mediated by influencing the cell cycle progression, flow cytometry was performed (Fig. 4A and 4B). Downregulation of SIRT1 led to a significant reduction in the number of BCPAP cells in the $\mathrm{G} 1$ phase and a significant accumulation of cells in $\mathrm{S}$ phase compared with the control (both $p<0.05$ ); moreover, when the upregulated SIRT1 expression was induced, fewer cells were hindered in S phase $(p<0.05)$. Consistent with these observations, SIRT1 overexpression facilitated cyclin D1 expression in BCPAP cells (Fig. 4C). No significant variations were observed in TPC-1 cells (Fig. 4B and 4D).

\section{SIRT1 reduced apoptosis in BRAF V600E-mutated cells}

Next, we performed a TUNEL assay to determine whether SIRT1 played a significant role in the apoptosis of TPC-1 and BCPAP cells. As shown in Fig. 5A, the percentage of BCPAP cells undergoing apoptosis was significantly increased in the SIRT1 siRNA group compared with that in the control cells at $48 \mathrm{~h}(p<0.01)$. In addition, the number of TUNEL-positive apoptotic cells in the SIRT1 overexpression group in BCPAP cells was significantly lower than that in the control group. However, we failed to observe any significant variations in TPC-1 cells (Fig. 5B). Consistently, the levels of apoptotic proteins, including BAX and BCL-2, in SIRT1-regulated TPC-1 and BCPAP cells were in agreement with the above results $(p<0.05$; Fig. $5 \mathrm{C}$ and $5 \mathrm{D})$. Therefore, SIRT1 is an important participant in the regulation of cell apoptosis in BCPAP cells.

\section{SIRT1 enhanced the migration and invasion potential of BRAF V600E-mutated cells}

Then, we investigated the functional role of SIRT1 in the migration and invasion of PTC cells. In wound healing assays, the distance of the scratch wound in the BCPAP SIRT1 siRNA group was significantly larger than that in the control group, whereas SIRT1 overexpression promoted the migration of BCPAP cells (both $p<0.01$; Fig. 6A). Nevertheless, no significant variations were observed in TPC-1 cells (Fig. 6B). Similar results were also found in the Matrigel invasion assay (Fig. 6C and 6D), in which the number of cells migrating through the chamber in the si-SIRT1 BCPAP group was significantly less than that in the control group $(p<0.01)$. In addition, the stimulatory effect of SIRT1 on BCPAP cell invasion was also confirmed $(p<0.01)$. However, we observed no significant variations in TPC-1 cells (Fig. 6D). These results indicated that SIRT1 enhanced the migration and invasion potential of BCPAP cells.

\section{SIRT1 facilitated phosphorylation of MAPK pathway proteins in BRAF V600E-mutated cells}

The above results showed that SIRT1 has different biological effects on TPC-1 and BCPAP cells. Since the distinct difference between these two cells is the BRAF V600E mutation status, we examined the activity of the MAPK signaling pathway. When SIRT1 was knocked down in BCPAP cells, the expression of $\mathrm{p}$-ERK1/2 and p-JNK (active forms) were significantly inhibited (both $p<0.01$ ), whereas those of their inactive forms (ERK1/2 and JNK) were unchanged (Fig. 7A). Thus, the loss of SIRT1 resulted in an overall 
reduced MAPK activity. In contrast, SIRT1 overexpression promoted their active forms in BCPAP cells, as confirmed by western blot analyses (Fig. 7A). However, no significant variations were found in TPC-1 cells (Fig. 7B). These data indicate that SIRT1 promoted the activity of ERK1/2 and JNK in the MAPK pathway and that SIRT1 is an important modulator of the MAPK pathway activity in BRAF V600E-mutated cells.

\section{Discussion}

Papillary thyroid cancer is the most common malignant tumor of the endocrine system, and the incidence has increased rapidly worldwide in recent years. The main treatment for PTC is surgery, radioactive iodine treatment and endocrine inhibition treatment. Most patients have a good prognosis. However, a small number of patients still show little or no effect under conventional treatments regardless of the tumor size or TMN, which could be due to their dedifferentiation status (28). In addition, the pathogenesis of PTC is complicated, and some patients possess a greater risk of advanced malignancy. Therefore, research on the pathogenesis and related regulatory molecules involved in PTC and on targeted intervention and treatment of these patients will help to improve their prognosis.

Here, we found that a higher SIRT1 expression in papillary thyroid cancer tissues and BCPAP cells than that in normal thyroid epithelial tissues and Nthy-ori $3-1$ cells, respectively. In addition, the BRAF V600E mutation facilitated SIRT1-promoted invasive growth and metastasis in PTC. We indicated that both SIRT1 and BRAF V600E mutations play synergistic roles in the development of papillary thyroid cancer. In this study, the results demonstrated the possibility that the BRAF V600E mutation in BCPAP cells mediates abnormal activation of the MAPK pathway.

BRAF mutations have been found in various tumors, such as melanoma, colorectal cancer, and thyroid cancer $(26,29,30)$. The disease-free survival and overall survival rates of BRAF mutant colorectal cancer patients were lower than those of unmutated wild-type BRAF patients $(31,32)$. In melanoma, patients with BRAF V600E mutations have a poor prognosis (27). Thus, BRAF V600E has been used as a molecular marker with diagnostic, prognostic, and predictive values for a variety of tumors $(15,33,34)$. As the most common mutation in PTC, BRAF V600E mutations are associated with large tumor lesions, multiple lesions, extraglandular infiltration, and lymph node metastasis, indicating that BRAF mutations are closely associated with poor prognosis of PTC $(35,36)$. Moreover, BRAF mutations are the most efficient way to activate the MAPK signaling pathway. BRAF V600E mutations could activate the BRAF kinase and downstream MAPK pathways (37). Verofenib, a selective BRAF inhibitor that is effective in treating BRAF V600E-mutant PTCs, suggests that thyroid cancer can be effectively treated by inhibiting BRAF kinase activation and lowering MAPK pathway activity (38). Many studies have confirmed that increased activation of the MAPK pathway is ubiquitously associated with BRAF mutations in thyroid cancer $(39,40)$.

SIRT1 plays an important role in the tumorigenesis, invasion and metastasis through the regulation of histones, non-histone proteins and other transcription factors $(41,42)$. Studies have shown that SIRT1 overexpression in Pten+/- mice leads to the development of thyroid tumors (43). Our study also confirmed 
that SIRT1 mRNA and protein expression levels were significantly increased in PTC, and the expression was related to the BRAF V600E mutation. Multiple studies have shown that SIRT1 overexpression is associated with poor prognosis in patients with clinical papillary thyroid carcinoma $(25,44)$, but the specific causes and mechanisms have not been reported. This study explored the correlation between SIRT1 and BRAF V600E mutations and the synergistic effect of those two mutations on PTC, which could be very helpful for early diagnosis, individualized treatment and prognosis of PTC. The above experiments confirmed that the effect of SIRT1 on BRAF V600E mutant PTC cells may be related to the abnormal activation of ERK and JNK/MAPK pathways by the BRAF V600E mutation by regulating the expression of SIRT1 in thyroid papillary carcinoma cells.

The MAPK pathway is an important signaling pathway. It can induce a variety of biological reactions, including cell proliferation, differentiation and apoptosis. Overexpression of various oncoproteins, such as chemokines and repressor proteins, was also reported to play a pivotal role in malignant transformation of cells $(45,46)$. MAPK/ERK and MAPK/JNK have been currently found in mammals. The MAPK pathway is regulated by upstream factors, including receptor tyrosine kinases (RTKs), RAS oncogenes, and RAF protein-serine/threonine kinases. Tyrosine kinase inhibitors (TKIs), including sorafenib, pazopanib, not only inhibit the MAPK pathway but also other target molecules and have been considered the most promising drug for the treatment of refractory thyroid cancer. $(47,48)$. Sorafenib was approved by the US Food and Drug Administration (FDA) for targeted treatment of radioactive iodinerefractory DTC (49). Although this experiment involves many aspects, we should increase the number of frozen specimens and paraffin tissue sections to minimize errors and deviations. In our further studies, we should include nodules that are less than one centimeter that affect the normal clinical pathological diagnosis at the same time.

Taken together, this study demonstrated for the first time that the SIRT1 expression is associated with the BRAF V600E mutation in PTC and that inhibition of SIRT1 could significantly weaken the proliferation, apoptosis, migration, and invasion of BRAF V600E mutant cells. Furthermore, when cells contain the BRAF V600E mutation, the MAPK pathway may play a pivotal role in regulating aggressive features of the cells. This study may help provide new therapeutic targets for clinical research and practice of PTC. The BRAF V600E mutation status combined with SIRT1 expression levels may be promising reference indicators for PTC diagnosis, prognosis, and development of treatment options.

\section{Abbreviations}

PTC, Papillary thyroid cancer; SIRT1, Silent mating type information regulation 2 homolog-1; BRAF, $V$ Raf murine sarcoma viral oncogene homolog B; MAPK, Mitogen-activated protein kinase; FTC, Follicular thyroid cancer; PDTC, Poorly differentiated thyroid cancer; ATC, Anaplastic thyroid cancer; TSH, Thyroidstimulating hormone; RAI, Radioactive iodine; DePTCs, Dedifferentiated papillary thyroid cancers; HIC-1, Hypermethylated in cancer-1; AJCC, American Joint Committee on Cancer; PVDF, Polyvinylidene fluoride; HRP, Horseradish peroxidase; FFPE, Formalin-fixed paraffin-embedded; siRNA, Small interfering RNA; NC, 
Negative control; TUNEL, Terminal deoxynucleotidyl transferase UTP nick-end labeling; ANT, adjacent normal tissues.

\section{Declarations}

- Ethics approval and consent to participate: The project protocols were approved by the Medical Ethics Committee of Second Hospital of Dalian Medical University.

- Consent for publication: All patients provided written informed consent for the use of their tumor tissues for clinical research.

- Availability of data and material: All data generated or analyzed during this study are included in this published article.

- Competing interests: The authors declare that they have no competing interests.

- Funding: Not applicable

- Authors' contributions: TL performed the laboratory experiments, drafted this manuscript and was a major contributor in writing the manuscript. GW and NZ contributed analysis tools and edited the manuscript. YL and $Y Z$ contributed analysis tools. XT conceived and designed the study, supervision and funding acquisition. All authors have read and approved the final manuscript.

- Acknowledgements: Not applicable

\section{References}

1. Miller KD, Siegel RL, Lin CC, Mariotto AB, Kramer JL, Rowland JH, et al. Cancer treatment and survivorship statistics, 2016. CA Cancer J Clin. 2016;66(4):271-89.

2. Pellegriti G, Frasca F, Regalbuto C, Squatrito S, Vigneri R. Worldwide increasing incidence of thyroid cancer: update on epidemiology and risk factors. J Cancer Epidemiol. 2013;2013:965212.

3. Roman BR, Morris LG, Davies L. The thyroid cancer epidemic, 2017 perspective. Curr Opin Endocrinol Diabetes Obes. 2017;24(5):332-6.

4. Hasbek Z, Turgut B, Erselcan T. p53 antibody: is it an indicator of dedifferentiated thyroid cancer? Ann Nucl Med. 2014;28(1):42-6.

5. Fallahi P, Mazzi V, Vita R, Ferrari SM, Materazzi G, Galleri D, et al. New therapies for dedifferentiated papillary thyroid cancer. Int J Mol Sci. 2015;16(3):6153-82.

6. Kelly LM, Barila G, Liu P, Evdokimova VN, Trivedi S, Panebianco F, et al. Identification of the transforming STRN-ALK fusion as a potential therapeutic target in the aggressive forms of thyroid cancer. Proc Natl Acad Sci U S A. 2014;111(11):4233-8.

7. Kimura ET, Nikiforova MN, Zhu Z, Knauf JA, Nikiforov YE, Fagin JA. High prevalence of BRAF mutations in thyroid cancer: genetic evidence for constitutive activation of the RET/PTC-RAS-BRAF signaling pathway in papillary thyroid carcinoma. Cancer Res. 2003;63(7):1454-7. 
8. Chapman PB, Hauschild A, Robert C, Haanen JB, Ascierto P, Larkin J, et al. Improved survival with vemurafenib in melanoma with BRAF V600E mutation. N Engl J Med. 2011;364(26):2507-16.

9. Barras D, Missiaglia E, Wirapati P, Sieber OM, Jorissen RN, Love C, et al. BRAF V600E Mutant Colorectal Cancer Subtypes Based on Gene Expression. Clin Cancer Res. 2017;23(1):104-15.

10. Xing $M$, Haugen BR, Schlumberger M. Progress in molecular-based management of differentiated thyroid cancer. Lancet. 2013;381(9871):1058-69.

11. Liu J, Liu R, Shen X, Zhu G, Li B, Xing M. The Genetic Duet of BRAF V600E and TERT Promoter Mutations Robustly Predicts the Loss of Radioiodine Avidity in Recurrent Papillary Thyroid Cancer. J Nucl Med. 2019.

12. Li XJ, Mao XD, Chen GF, Wang QF, Chu XQ, Hu X, et al. High BRAFV600E mutation frequency in Chinese patients with papillary thyroid carcinoma increases diagnostic efficacy in cytologically indeterminate thyroid nodules. Med (Baltim). 2019;98(28):e16343.

13. Neiswender JV, Kortum RL, Bourque C, Kasheta M, Zon LI, Morrison DK, et al. KIT Suppresses BRAF(V600E)-Mutant Melanoma by Attenuating Oncogenic RAS/MAPK Signaling. Cancer Res. 2017;77(21):5820-30.

14. Nakamura Y, Hattori N, lida N, Yamashita S, Mori A, Kimura K, et al. Targeting of super-enhancers and mutant BRAF can suppress growth of BRAF-mutant colon cancer cells via repression of MAPK signaling pathway. Cancer Lett. 2017;402:100-9.

15. Huang Y, Qu S, Zhu G, Wang F, Liu R, Shen X, et al. BRAF V600E Mutation-Assisted Risk Stratification of Solitary Intrathyroidal Papillary Thyroid Cancer for Precision Treatment. J Natl Cancer Inst. 2018;110(4):362-70.

16. Yan H, Jihong Y, Feng Z, Xiaomei X, Xiaohan Z, Guangzhi W, et al. Sirtuin 1-mediated inhibition of p66shc expression alleviates liver ischemia/reperfusion injury. Crit Care Med. 2014;42(5):e373-81.

17. Latifkar A, Ling L, Hingorani A, Johansen E, Clement A, Zhang X, et al. Loss of Sirtuin 1 Alters the Secretome of Breast Cancer Cells by Impairing Lysosomal Integrity. Dev Cell. 2019;49(3):393408.e7.

18. Lin Z, Fang D. The Roles of SIRT1 in Cancer. Genes Cancer. 2013;4(3-4):97-104.

19. Chen WY, Wang DH, Yen RC, Luo J, Gu W, Baylin SB. Tumor suppressor HIC1 directly regulates SIRT1 to modulate p53-dependent DNA-damage responses. Cell. 2005;123(3):437-48.

20. Lin Z, Yang H, Kong Q, Li J, Lee SM, Gao B, et al. USP22 antagonizes p53 transcriptional activation by deubiquitinating Sirt1 to suppress cell apoptosis and is required for mouse embryonic development. Mol Cell. 2012;46(4):484-94.

21. So D, Shin HW, Kim J, Lee M, Myeong J, Chun YS, et al. Cervical cancer is addicted to SIRT1 disarming the AIM2 antiviral defense. Oncogene. 2018;37(38):5191-204.

22. Yarahmadi S, Abdolvahabi Z, Hesari Z, Tavakoli-Yaraki M, Yousefi Z, Seiri P, et al. Inhibition of sirtuin 1 deacetylase by miR-211-5p provides a mechanism for the induction of cell death in breast cancer cells. Gene. 2019;711:143939. 
23. Choupani J, Mansoori Derakhshan S, Bayat S, Alivand MR, Shekari Khaniani M. Narrower insight to SIRT1 role in cancer: A potential therapeutic target to control epithelial-mesenchymal transition in cancer cells. J Cell Physiol. 2018;233(6):4443-57.

24. Ji K, Sun X, Liu Y, Du L, Wang Y, He N, et al. Regulation of Apoptosis and Radiation Sensitization in Lung Cancer Cells via the Sirt1/NF-kappaB/Smac Pathway. Cell Physiol Biochem. 2018;48(1):30416.

25. Wu W, Zhang L, Lin J, Huang H, Shi B, Lin X, et al. Hypermethylation of the HIC1 promoter and aberrant expression of HIC1/SIRT1 contribute to the development of thyroid papillary carcinoma. Oncotarget. 2016;7(51):84416-27.

26. Michaloglou C, Vredeveld LC, Soengas MS, Denoyelle C, Kuilman T, van der Horst CM, et al. BRAFE600-associated senescence-like cell cycle arrest of human naevi. Nature. 2005;436(7051):720-4.

27. Lito P, Pratilas CA, Joseph EW, Tadi M, Halilovic E, Zubrowski M, et al. Relief of profound feedback inhibition of mitogenic signaling by RAF inhibitors attenuates their activity in BRAFV600E melanomas. Cancer Cell. 2012;22(5):668-82.

28. Integrated genomic characterization. of papillary thyroid carcinoma. Cell. 2014;159(3):676-90.

29. Samowitz WS, Sweeney C, Herrick J, Albertsen H, Levin TR, Murtaugh MA, et al. Poor survival associated with the BRAF V600E mutation in microsatellite-stable colon cancers. Cancer Res. 2005;65(14):6063-9.

30. Xing M, Alzahrani AS, Carson KA, Viola D, Elisei R, Bendlova B, et al. Association between BRAF V600E mutation and mortality in patients with papillary thyroid cancer. Jama. 2013;309(14):1493501.

31. Damsky WE, Curley DP, Santhanakrishnan M, Rosenbaum LE, Platt JT, Gould Rothberg BE, et al. betacatenin signaling controls metastasis in Braf-activated Pten-deficient melanomas. Cancer Cell. 2011;20(6):741-54.

32. Di Nicolantonio F, Martini M, Molinari F, Sartore-Bianchi A, Arena S, Saletti P, et al. Wild-type BRAF is required for response to panitumumab or cetuximab in metastatic colorectal cancer. J Clin Oncol. 2008;26(35):5705-12.

33. Morikawa T, Inada R, Nagasaka T, Mori Y, Kishimoto H, Kawai T, et al. BRAF V600E mutation is a predictive indicator of upfront chemotherapy for stage IV colorectal cancer. Oncol Lett. 2018;15(2):2195-201.

34. VanderLaan PA, Rangachari D, Majid A, Parikh MS, Gangadharan SP, Kent MS, et al. Tumor biomarker testing in non-small-cell lung cancer: A decade of change. Lung Cancer. 2018;116:90-5.

35. Elisei R, Viola D, Torregrossa L, Giannini R, Romei C, Ugolini C, et al. The BRAF(V600E) mutation is an independent, poor prognostic factor for the outcome of patients with low-risk intrathyroid papillary thyroid carcinoma: single-institution results from a large cohort study. J Clin Endocrinol Metab. 2012;97(12):4390-8. 
36. Li C, Lee KC, Schneider EB, Zeiger MA. BRAF V600E mutation and its association with clinicopathological features of papillary thyroid cancer: a meta-analysis. J Clin Endocrinol Metab. 2012;97(12):4559-70.

37. Flaherty KT, Infante JR, Daud A, Gonzalez R, Kefford RF, Sosman J, et al. Combined BRAF and MEK inhibition in melanoma with BRAF V600 mutations. N Engl J Med. 2012;367(18):1694-703.

38. Kim KB, Cabanillas ME, Lazar AJ, Williams MD, Sanders DL, llagan JL, et al. Clinical responses to vemurafenib in patients with metastatic papillary thyroid cancer harboring BRAF(V600E) mutation. Thyroid. 2013;23(10):1277-83.

39. Zou M, Baitei EY, BinEssa HA, Al-Mohanna FA, Parhar RS, St-Arnaud R, et al. Cyp24a1 Attenuation Limits Progression of Braf(V600E) -Induced Papillary Thyroid Cancer Cells and Sensitizes Them to BRAF(V600E) Inhibitor PLX4720. Cancer Res. 2017;77(8):2161-72.

40. Nagarajah J, Le M, Knauf JA, Ferrandino G, Montero-Conde C, Pillarsetty N, et al. Sustained ERK inhibition maximizes responses of BrafV600E thyroid cancers to radioiodine. J Clin Invest. 2016;126(11):4119-24.

41. Strzyz P. Stress responses: SIRT1 puts an embargo on mRNA export. Nat Rev Mol Cell Biol. 2017;18(9):530-1.

42. Imperatore F, Maurizio J, Vargas Aguilar S, Busch CJ, Favret J, Kowenz-Leutz E, et al. SIRT1 regulates macrophage self-renewal. Embo j. 2017;36(16):2353-72.

43. Herranz D, Maraver A, Canamero M, Gomez-Lopez G, Inglada-Perez L, Robledo M, et al. SIRT1 promotes thyroid carcinogenesis driven by PTEN deficiency. Oncogene. 2013;32(34):4052-6.

44. Wang C, Yang W, Dong F, Guo Y, Tan J, Ruan S, et al. The prognostic role of Sirt1 expression in solid malignancies: a meta-analysis. Oncotarget. 2017;8(39):66343-51.

45. Lee HJ, Im AR, Kim SM, Kang HS, Lee JD, Chae S. The flavonoid hesperidin exerts anti-photoaging effect by downregulating matrix metalloproteinase (MMP)-9 expression via mitogen activated protein kinase (MAPK)-dependent signaling pathways. BMC Complement Altern Med. 2018;18(1):39.

46. Song ZY, Wang F, Cui SX, Qu XJ. Knockdown of CXCR4 Inhibits CXCL12-Induced Angiogenesis in HUVECs through Downregulation of the MAPK/ERK and PI3K/AKT and the Wnt/beta-Catenin Pathways. Cancer Invest. 2018;36(1):10-8.

47. Bible KC, Suman VJ, Menefee ME, Smallridge RC, Molina JR, Maples WJ, et al. A multiinstitutional phase 2 trial of pazopanib monotherapy in advanced anaplastic thyroid cancer. $\mathrm{J}$ Clin Endocrinol Metab. 2012;97(9):3179-84.

48. Schneider DF, Chen H. New developments in the diagnosis and treatment of thyroid cancer. CA Cancer J Clin. 2013;63(6):374-94.

49. Gupta-Abramson V, Troxel AB, Nellore A, Puttaswamy K, Redlinger M, Ransone K, et al. Phase II trial of sorafenib in advanced thyroid cancer. J Clin Oncol. 2008;26(29):4714-9.

\section{Tables}


Table 1: Association between SIRT1 expression and clinicopathological features of papillary thyroid cancer (PTC) patients.

\begin{tabular}{|c|c|c|c|c|c|}
\hline \multirow[t]{2}{*}{ Variables } & \multirow[t]{2}{*}{ No. of cases } & \multicolumn{2}{|c|}{ SIRT1 expression } & \multirow[t]{2}{*}{$\chi^{2}$} & \multirow[t]{2}{*}{ P-value } \\
\hline & & Low & High & & \\
\hline Age (years) & & & & 0.6841 & $>0.05$ \\
\hline$<55$ & 17 & 9 & 8 & & \\
\hline$\geq 55$ & 25 & 10 & 15 & & \\
\hline Gender & & & & 0.006373 & $>0.05$ \\
\hline Male & 13 & 6 & 7 & & \\
\hline Female & 29 & 13 & 16 & & \\
\hline Tumor size (cm) & & & & 0.08009 & $>0.05$ \\
\hline$<1.5$ & 24 & 11 & 13 & & \\
\hline$\geq 1.5$ & 18 & 8 & 10 & & \\
\hline TNM stage & & & & 0.2310 & $>0.05$ \\
\hline I-II & 26 & 12 & 14 & & \\
\hline III-IV & 16 & 7 & 9 & & \\
\hline LN metastasis & & & & 6.402 & $<0.05$ \\
\hline No & 10 & 8 & 2 & & \\
\hline Yes & 32 & 11 & 21 & & \\
\hline BRAF V600E mutation & & & & 8.050 & $<0.01$ \\
\hline No & 11 & 9 & 2 & & \\
\hline Yes & 31 & 10 & 21 & & \\
\hline
\end{tabular}

Table 2: Association between BRAF V600E mutation and lymph node metastasis of PTC patients.

\begin{tabular}{lccccc}
\hline Variable & No. of cases & BRAF V600E mutation & \multirow{2}{*}{$\chi^{2}$} & P-value \\
\cline { 3 - 4 } & & Non-mutant & Mutant & & \\
\hline LN metastasis & 10 & 5 & 5 & & \\
No & 32 & 6 & 26 & & \\
Yes & & & & & \\
\hline
\end{tabular}

Figures 
A

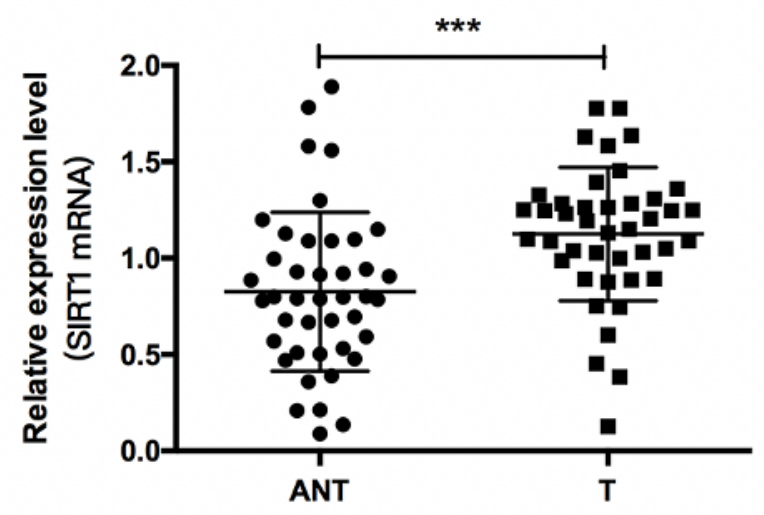

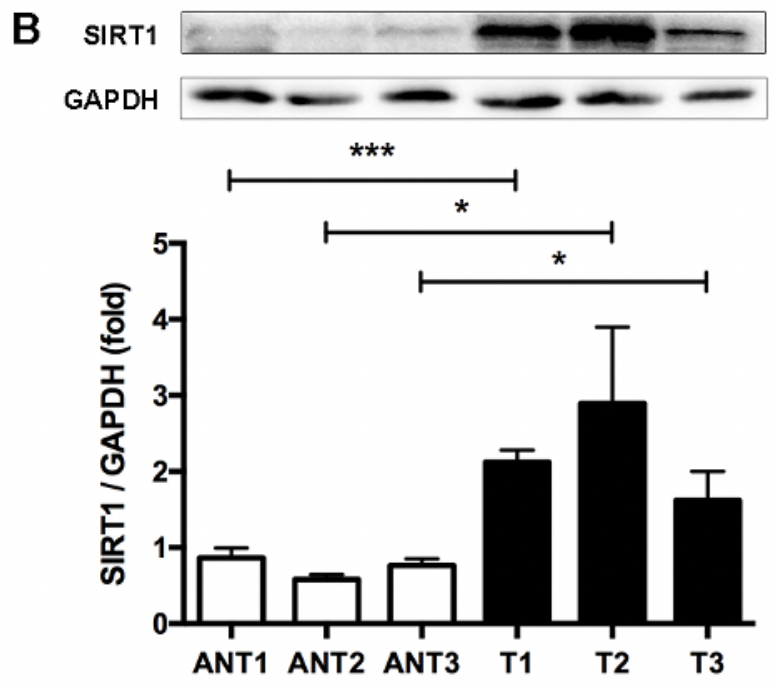

\section{Figure 1}

SIRT1 expression is upregulated in PTC tissues. (A) Real-time quantitative RT-PCR (qRT-PCR) analyses of SIRT1 expression in PTC tissues (T) and their adjacent normal tissues (ANTs). (B) Western blotting analyses of SIRT1 expression in three representative pairs of PTC tissues and their ANTs. ${ }^{*} p<0.05$ and *** $p<0.001$ versus ANT group. 

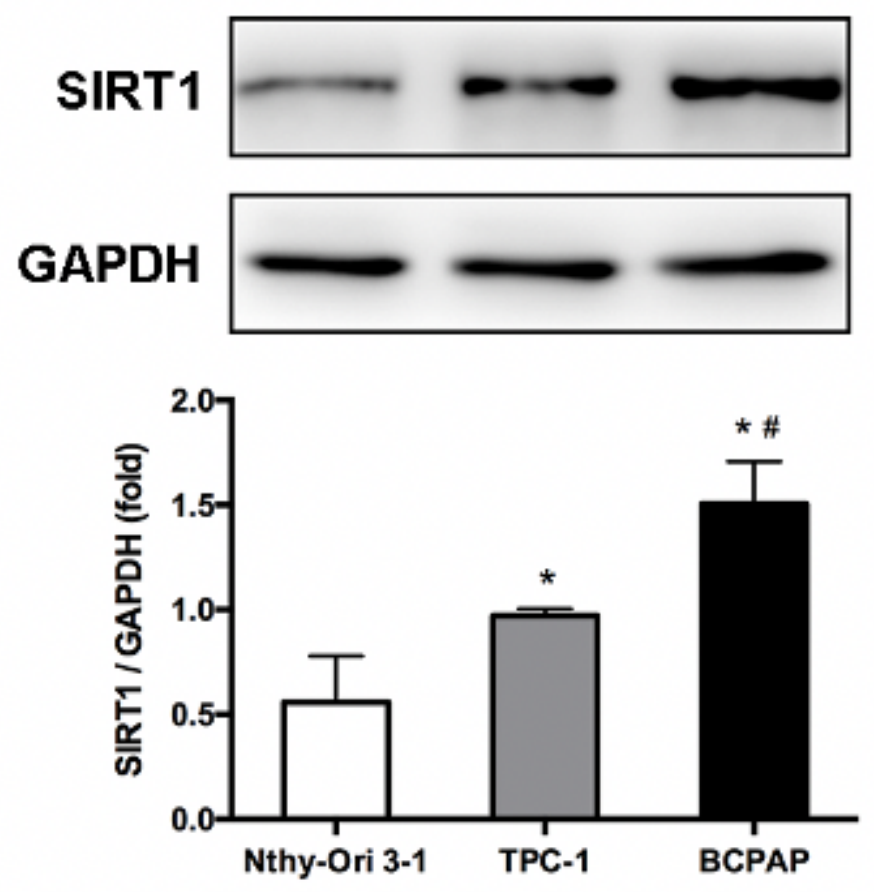

Figure 2

BRAF V600E mutated PTC cells possessed the highest level of SIRT1 expression. Western blotting analysis of SIRT1 expression in TPC-1 and BCPAP papillary thyroid cancer cell lines compared with one normal thyroid follicular epithelial cell line. ${ }^{*} p<0.05$ versus Nthy-Ori 3-1 group; $\# p<0.05$ versus TPC-1 group. 
A
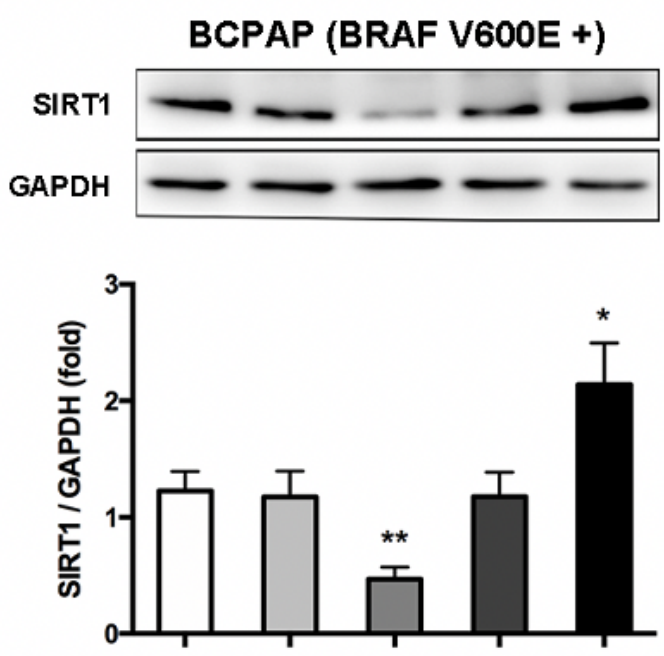

Control si-NC Si-SIRT1

CRISPR NC CRISPR SIRT1

C

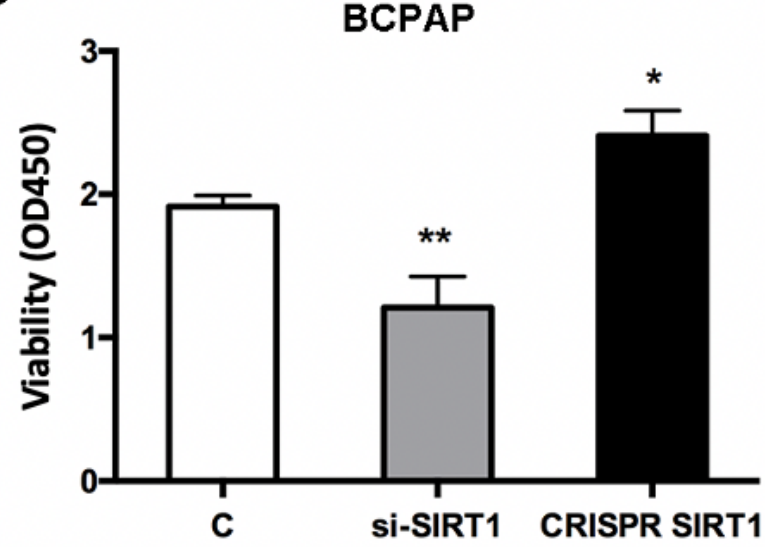

B TPC-1 (BRAF V600E-)
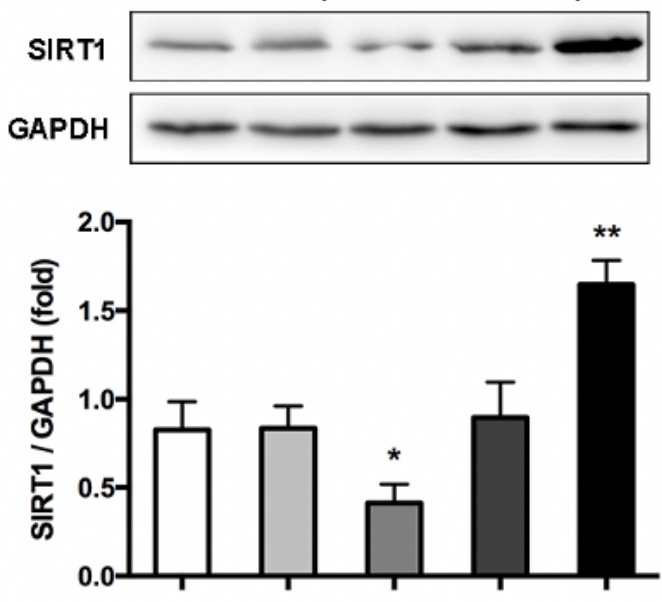

Control si-NC Si-SIRT1

CRISPR NC CRISPR SIRT1

D

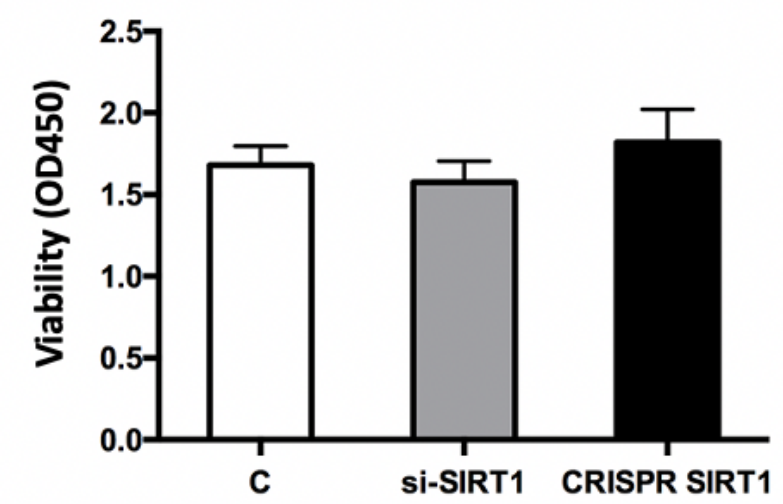

Figure 3

SIRT1 promoted cell proliferation of BRAF V600E mutated PTC cells. (A and B) Western blotting analyses of SIRT1 expression following the treatment of TPC-1 and BCPAP cells with si-SIRT1, CRISPR SIRT1, and respectively negative control vectors. (C and D) CCK-8 assays were performed to evaluated TPC- 1 and BCPAP cell viability after transfection with SIRT1-specific siRNAs and CRISPR SIRT1 activation plasmids. $\star p<0.05 ; * \star p<0.01$. 

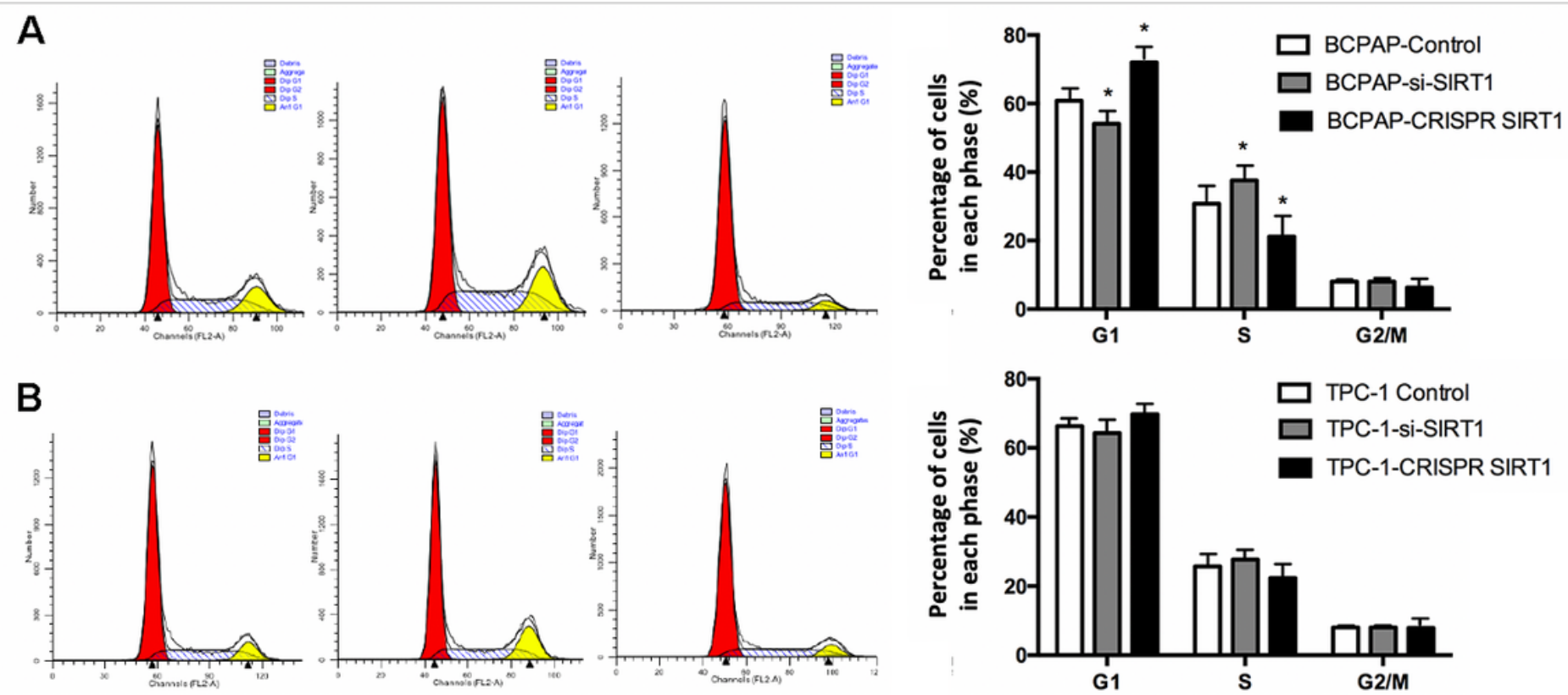

C

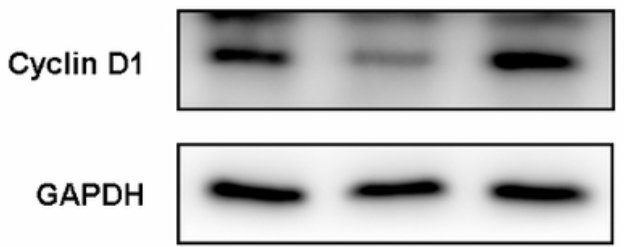

D
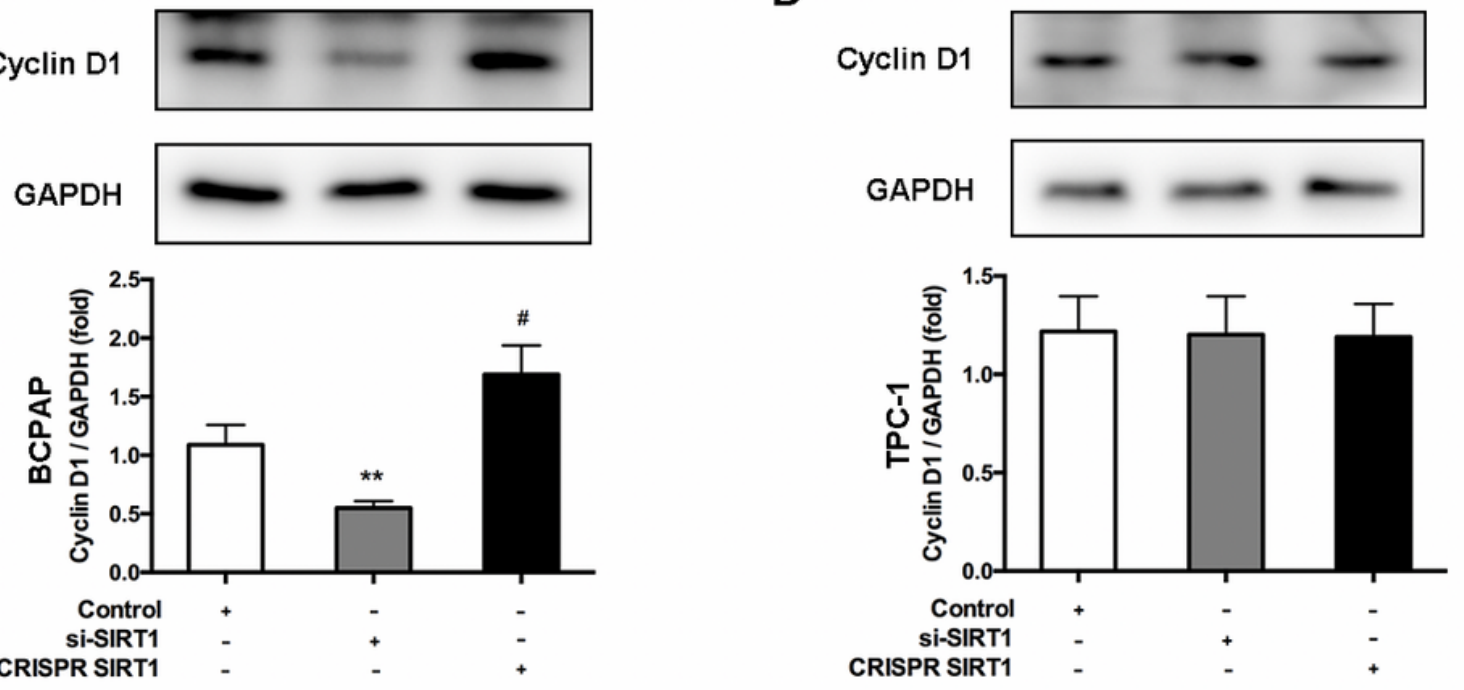

Figure 4

SIRT1 facilitated cell cycle progression in BRAF V600E mutated PTC cells. TPC-1 and BCPAP were treated with si-SIRT1 of CRISPR SIRT1 plasmids. (A and B) Flow cytometry were used to analyze cell cycle distributions of PTC cells. (C and D) Western blotting analyses of Cyclin D1 expression in TPC-1 and BCPAP. ${ }^{*} p<0.05 ; * \star p<0.01$. 
A

Control

si-SIRT1
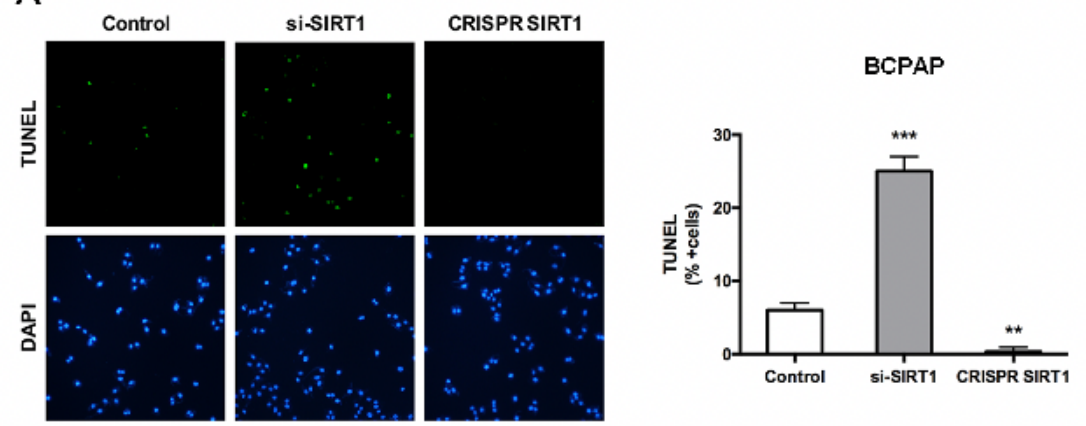

B
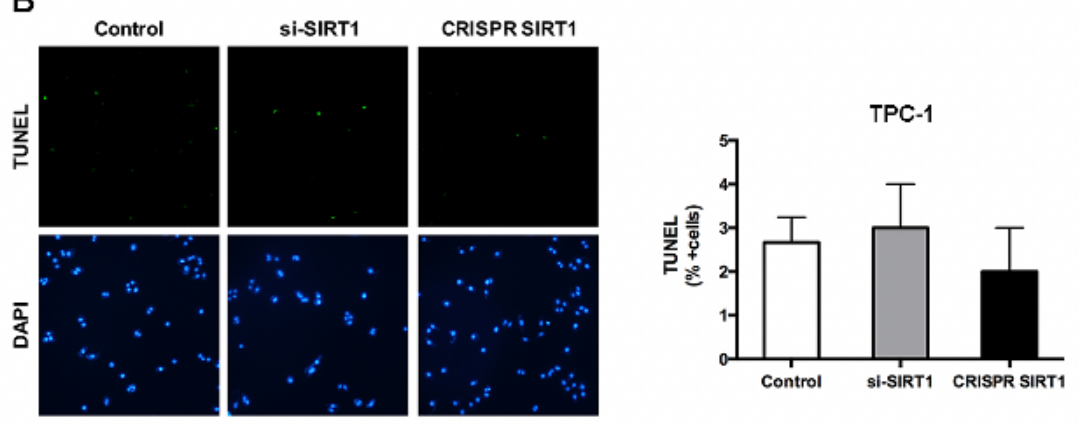

C
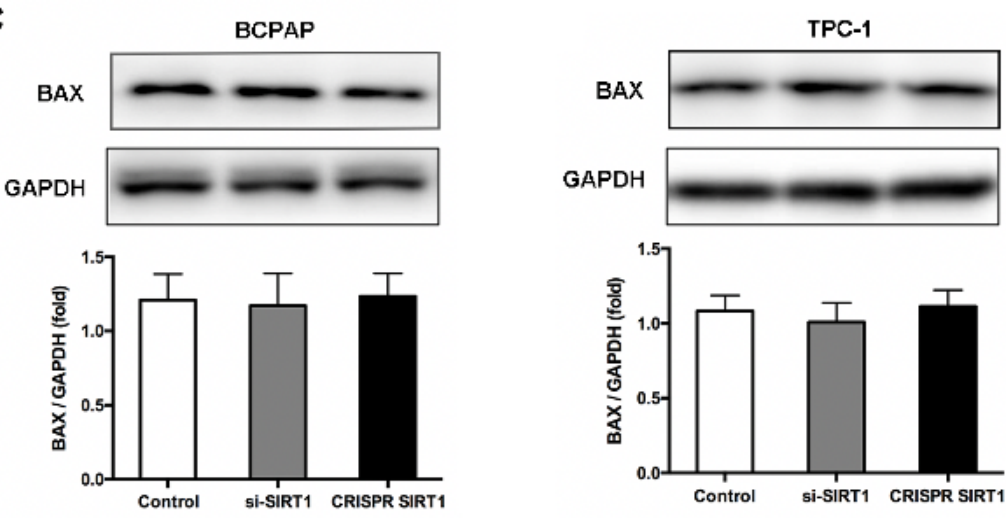

D
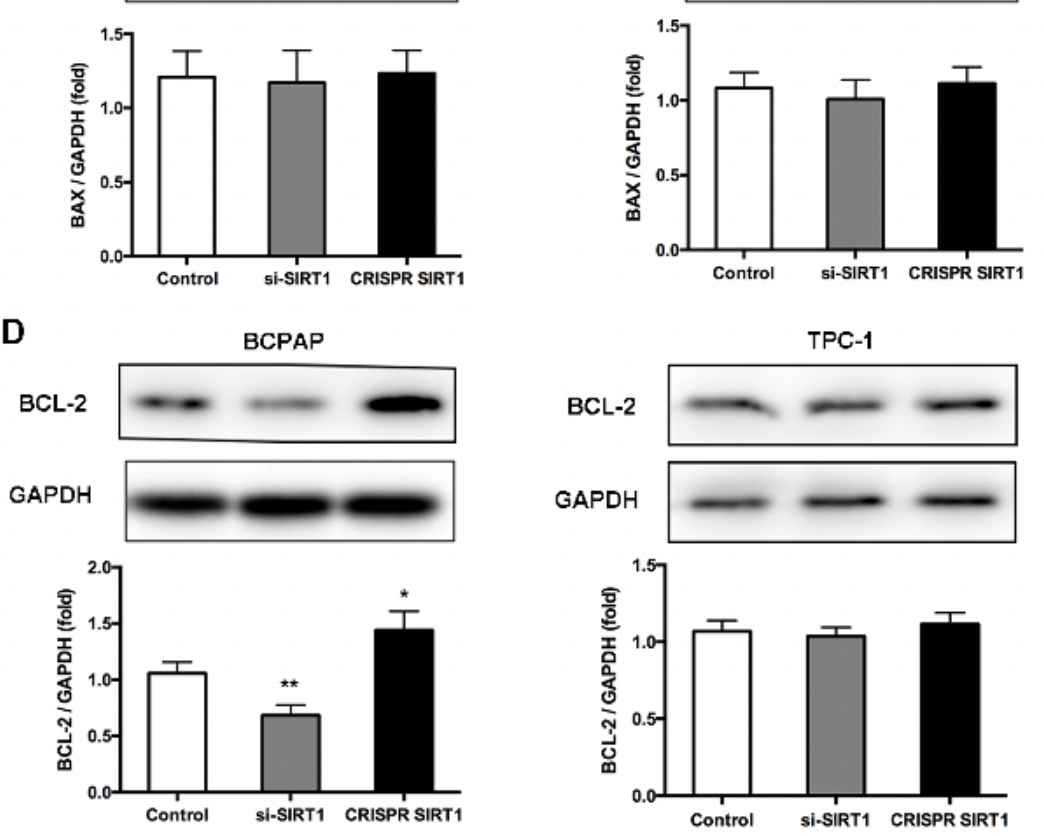

\section{Figure 5}

SIRT1 reduced apoptosis in BRAF V600E mutated PTC cells. TPC-1 and BCPAP were treated with si-SIRT1 of CRISPR SIRT1 plasmids. (A and B) Representative fluorescent images of TUNEL-positive (green) and DAPI (blue) staining PTC cells at $48 \mathrm{~h}$ after treatment. Scale bar=100 $\mu \mathrm{m}$. (C and D) Western blotting analyses of apoptotic protein (BAX and BCL-2) expression in BCPAP and TPC- 1. * $p<0.05 ;$ ** $p<0.01$; $\star \star \star ~ p<0.001$. 


\section{Figure 6}

SIRT1 enhanced the migration and invasion potential of BRAF V600E mutated PTC cells. TPC-1 and BCPAP were treated with si-SIRT1 of CRISPR SIRT1 plasmids. ( $A$ and B) Wound healing assays showed that SIRT1 promotes migration in BCPAP cells. (C-D) Matrigel invasion assays showed that SIRT1 only enhances invasion ability of BCPAP cells. ${ }^{* *} p<0.01$.

A

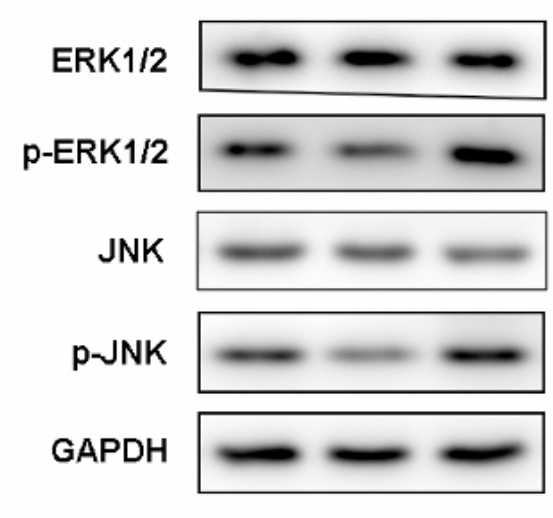

B

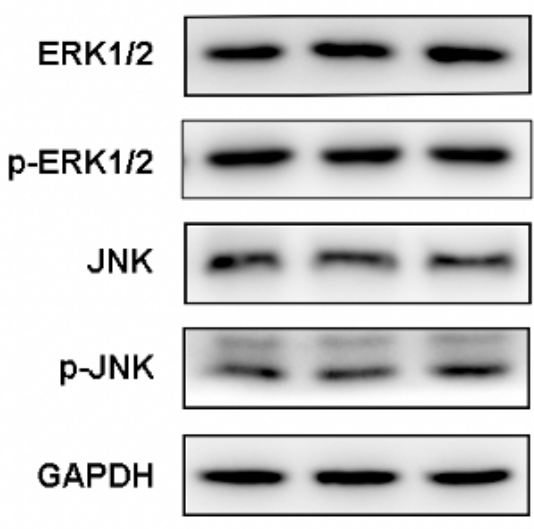

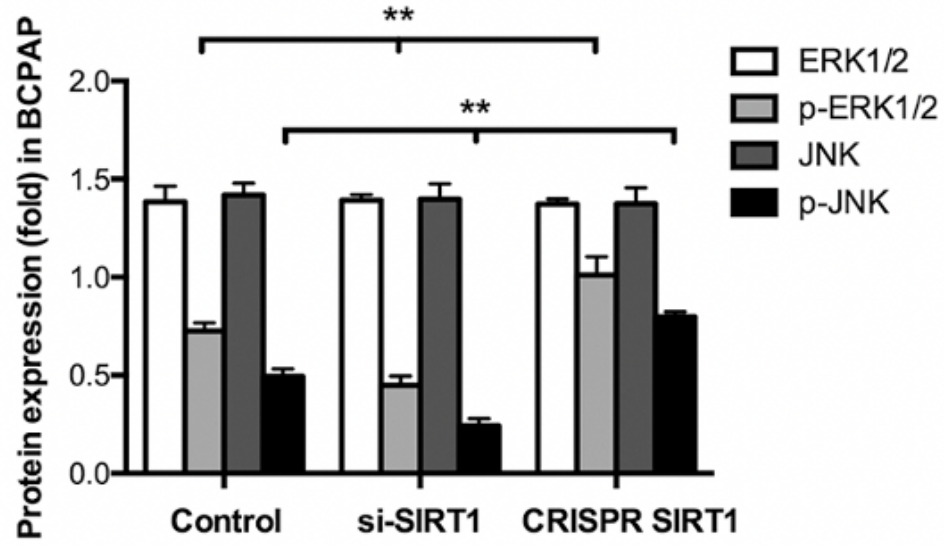

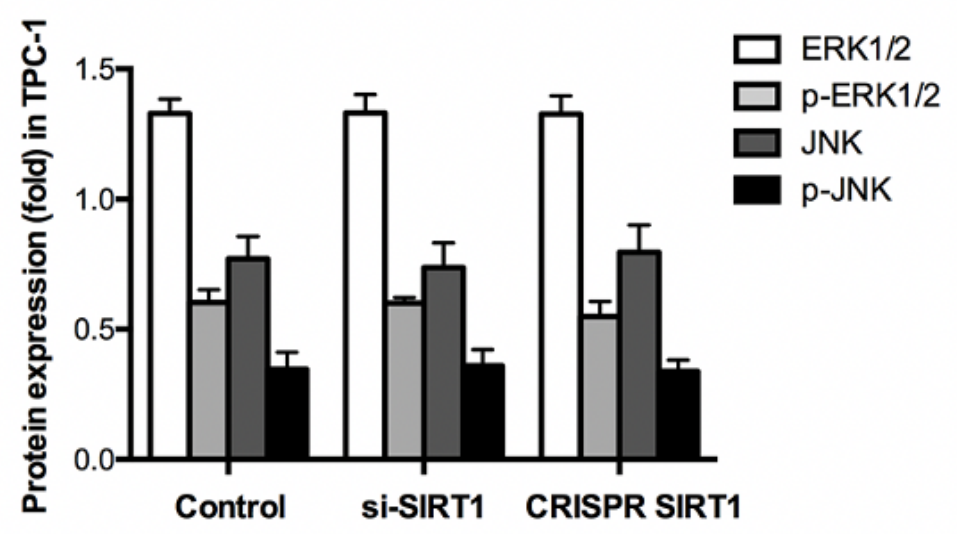

Figure 7

SIRT1 facilitated activation of MAPK pathway in BRAF V600E mutated PTC cells. TPC-1 and BCPAP were treated with si-SIRT1 of CRISPR SIRT1 plasmids. The expression of ERK1/2 and JNK MAPK proteins were investigated in BCPAP (A) and TPC-1 (B) cells. ${ }^{* *} p<0.01$. All the experiments were performed in triplicate. 